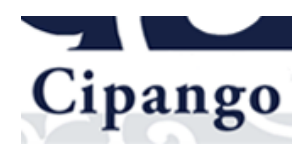

\title{
Cipango
}

Cahiers d'études japonaises

16 | 2009

L'invention des « arts populaires » - Yanagi Sōetsu et le Mingei

\section{Folk art et folk studies}

Débat entre Yanagita Kunio et Yanagi Sōetsu, à la Maison des arts populaires

Folk Crafts and Folklore Studies

Kunio Yanagita, Sōetsu Yanagi et Damien Kunik

Traducteur : Damien Kunik et Jean-Michel Butel

\section{(2) OpenEdition}

Édition électronique

URL : https://journals.openedition.org/cipango/355

DOI : $10.4000 /$ cipango.355

ISSN : 2260-7706

Éditeur

INALCO

Édition imprimée

Date de publication : 1 janvier 2009

Pagination : 105-120

ISSN : 1164-5857

Référence électronique

Kunio Yanagita, Sōetsu Yanagi et Damien Kunik, « Folk art et folk studies », Cipango [En ligne], 16 |

2009, mis en ligne le 18 décembre 2011, consulté le 30 juin 2021. URL : http://

journals.openedition.org/cipango/355; DOI : https://doi.org/10.4000/cipango.355

Ce document a été généré automatiquement le 30 juin 2021.

\section{(c) (1) (9)}

Cipango est mis à disposition selon les termes de la Licence Creative Commons Attribution - Pas d'Utilisation Commerciale 4.0 International. 


\section{Folk art et folk studies}

Débat entre Yanagita Kunio et Yanagi Sōetsu, à la Maison des arts populaires

Folk Crafts and Folklore Studies

Kunio Yanagita, Sōetsu Yanagi et Damien Kunik

Traduction : Damien Kunik et Jean-Michel Butel

\section{Présentation du débat par Damien Kunik}

1 Entre 1938 et 1940, Yanagi Sōetsu, accompagné de plusieurs membres de l'Association pour les arts populaires, fait trois voyages à Okinawa avec pour objectif d'y étudier l'artisanat local. Agacé par la politique assimilationniste des autorités japonaises sur place, il se prend à défendre les particularismes locaux et le patrimoine régional d'Okinawa. Le débat très animé, qui porte essentiellement sur la question de la préservation des dialectes de ces îles mis à mal par la promotion d'une langue japonaise standardisée, fera plusieurs fois la une des journaux durant ces années.

2 Au début de l'année 1940, Yanagi, devenu en quelque sorte le "spécialiste d'Okinawa", est approché par la NHK pour débattre, lors d'un entretien radiophonique, avec un autre scientifique qui s'était intéressé à la question des Ryūkyū, Yanagita Kunio.

3 Yanagi et Yanagita, qui se rencontrent pour la première fois à cette occasion, se connaissent mutuellement par le biais de leurs publications. Or, ce n'est pas tant la question d'Okinawa qui les intéresse, mais plutôt les distinctions entre les approches dont ils sont, l'un et l'autre, les chefs de file. Ainsi, le débat sur Okinawa est largement mis de côté au profit d'une discussion sur la nature de leurs méthodes respectives. C'est la traduction de cette partie de l'entretien que nous donnons ici.

4 Si Yanagi et Yanagita n'ont nullement besoin d'être introduits, nous souhaitons en revanche présenter Shikiba Ryūzaburō 式場隆三郎 (1898- 1965), troisième acteur du débat, dont l'importance au sein du mouvement des Arts populaires durant les années 1930-1940 est extrêmement sous-estimée. Shikiba, médecin et psychiatre de métier, est également un écrivain prolifique et grand amateur de peinture. Traducteur et commentateur du Marquis de Sade, 
rédacteur de plusieurs livres sur l'éducation sexuelle des jeunes filles, protecteur du «Van Gogh japonais » Yamashita Kiyoshi 山下清 (1922-1971), Shikiba marque de sa personnalité contrastée le mouvement des Arts populaires. Dès 1939, il codirige, avec Yoshida Shōya, le Mensuel des arts populaires (Gekkan Mingei 月刊民藝), la 《mitraillett $e^{1}$ " du mouvement. Son militantisme, très utile à la diffusion de l'idéologie des Arts populaires auprès du gouvernement Konoe durant ces années, sera discrètement oublié après 1945. Après-guerre, Shikiba fondera les organes de presse Tokyo Times et Romance, ce dernier relevant plutôt de la presse à sensation. Son omniprésence au sein du mouvement avant la fin de la guerre explique cependant sans surprise son apparition au sein du débat présenté ici, qui a priori ne le concerne pas.

Nous choisissons de traduire cet entretien dans l'espoir que le lecteur pourra y relever l'illustration d'éléments constitutifs du mouvement pour les Arts populaires largement méconnus, comme son besoin de définition de la discipline et d'affirmation identitaire, ou ses préoccupations mercantilistes par exemple. Nous espérons ainsi offrir une alternative au discours consensuel sur le mouvement de Yanagi, qui fait de celui-ci un courant d'art décoratif à la philosophie éthérée. Comme le lecteur pourra le constater, il n'y a rien de tout cela dans le quotidien du mouvement, qui se préoccupe plus du succès de ses expositions ou de la diffusion de ses produits ${ }^{2}$.

\section{Folk art et folk studies}

Débat entre Yanagita Kunio et Yanagi Sōetsu, à la Maison des arts populaires ${ }^{3}$, en présence de Higa Shunchō, du département d'okinawa.

Modérateur : Ryūzaburō Shikiba

\section{Qu'appelle-t-on « études folkloriques »?}

Shikiba : Permettez-moi d'introduire le débat de ce jour. Le mouvement pour les Arts populaires que nous animons actuellement à la Maison des arts populaires [Mingeikan 民藝館], et les études folkloriques du professeur Yanagita Kunio, sont très souvent confondus. Il nous a donc semblé important de réunir messieurs Yanagita et Yanagi afin de tenter de fixer clairement leurs différences. Cela pourrait d'ailleurs être bénéfique aux deux disciplines.

YANAgı: J'aurais aimé savoir quand ont pris leur essor les études des mœurs autochtones ${ }^{4}$ ?

Yanagita : Elles sont en vérité antérieures aux débuts de la psychologie. On peut les rapprocher de l'ethnologie $\mathrm{e}^{5} \mathrm{il}$ me semble.

YANAgI : Ces études attirent-elles toujours l'attention aux États-Unis ou en Angleterre?

Yanagita : L'intérêt pour l'étude des mœurs nationales n'y est pas très fort. On met en effet plutôt l'accent généralement sur l'étude des groupes ethniques ${ }^{6}$.

SHIкıвA : Professeur Yanagita, comment faut-il appeler ce que vous pratiquez ? Étude des mœurs autochtones [dozokugaku] ? des mœurs populaires [minzokugaku 民俗学] ?

Yanagita : Ce terme d'« étude des mœurs autochtones » est en fait très gênant pour nous. Le mot évoque en effet, d'une certaine manière, celui d'aborigène ${ }^{7}$. Voilà pourquoi nous évitons de l'utiliser et lui préférons celui d'« études folkloriques". Terme qui n'est pas sans poser problème à son tour, puisqu'il est homonyme de celui d'ethnologie ${ }^{8}$. 
YANAgı: L'ethnologie se préoccupe essentiellement, si je comprends bien, des peuples primitifs $^{9}$ ?

Yanagita : Oui, des peuples non développés. Ou semi-développés ${ }^{10}$. Cependant, aujourd'hui, au Japon, il n'est plus possible de pratiquer cette recherche autrement qu'en consultant des livres. Or, si elles peuvent, à leurs heures, consulter des documents écrits, nos études folkloriques s'appuient d'abord sur l'observation directe. Ainsi, les méthodes diffèrent dans leur principe même.

Yanagı : Ōyama Kashiwa, par exemple, parle, pour ce qu'il fait, d'études " préhistoriques ${ }^{11} »$ je crois.

Yanagita : À vrai dire, ses travaux relèvent plutôt de l'anthropologie. On peut distinguer anthropologie physique et anthropologie culturelle $\mathrm{e}^{12}$; il me semble que ce dont vous parlez relève plutôt de l'anthropologie culturelle. Comme il s'agit avant tout d'une étude matérielle, qui se soucie peu d'établir une distinction entre étude du pays auquel appartient le chercheur et étude d'un pays autre, je pense que cela est très proche de l'archéologie ${ }^{13}$. C'est donc très différent de notre projet.

SHIKıвA : Lorsque l'on lit vos ouvrages, professeur Yanagita, on y trouve de nombreuses références à la langue et au lexique. S'agit-il d'une posture méthodologique?

Yanagita : Il y a de nombreuses raisons à cela, la première étant que j'ai une grande passion pour les mots et que je me laisse sans doute emporter par mon intérêt pour la linguistique ${ }^{14}$ ! Mais cela est aussi bien pratique en tant que méthode. On aimerait avoir toujours des choses concrètes à étudier, mais il arrive que ce ne soit pas le cas. Or on peut faire toutes sortes de découvertes grâce à un mot, quand on s'intéresse précisément à son contexte. Cependant, il est très facile de se tromper avec les mots, quand on n'y fait pas suffisamment attention. Il est donc nécessaire de prendre les précautions adéquates... En réalité, nous devrions, nous aussi, observer des objets concrets un peu plus que nous ne le faisons habituellement, mais ce faisant nous risquerions de nous bloquer sur ce type de recherche... Alors pour l'instant nous nous contentons de traiter d'illustrations, ou de photographies.

SHוкıвA : Cependant, ne pourrait-on pas penser que, du point de vue de la méthode, une étude ne saurait être complète sans la prise en compte d'objets réels, tels ceux relevant de l'art populaire ${ }^{15}$ ?

Yanagita : Prenons, si vous le voulez bien, l'exemple des transportations ${ }^{16}$, de ce qui permet de transporter des choses. Afin d'en faire l'étude, il faut bien sûr analyser concrètement les différents types de paniers, par exemple. Maintenant que nous avons des trains et des voitures, il n'en reste plus grand chose, mais jusqu'à très récemment étaient utilisés de nombreux paniers. Or les palanches qui servaient à porter ces paniers diffèrent grandement selon qu'elles ont ou non une protubérance en leurs extrémités, l'absence de protubérance obligeant en effet à effectuer un nœud particulier, notamment. Il y avait ainsi toutes sortes de perches. Nous aimerions qu'on nous montre ces objets de façon organisée. Je ne pense pas que l'on réunisse ces outils à la Maison des arts populaires, mais c'est quelque chose que l'on peut confier à l'Attic Museum de M. Shibusawa Keizō ${ }^{17}$. Je ne sais pas quel est vraiment son projet mais il me semble que son approche penche du côté du folklore. Ce point constitue une différence certaine entre la Maison des arts populaires et l'Attic Museum. 
YANAgI: II est vrai qu'il n'est pas dans l'intention de la Maison des arts populaires de collectionner de la sorte tous les objets anciens. Nous avons plutôt comme politique de ne collecter que des pièces d'artisanat populaire belles et justes ${ }^{18}$.

Yanagita : De mon côté, j'essaie plutôt, sur le plan de la méthode, de mettre des forces d'abord sur le point suivant: la culture n'avance pas de front, comme une armée en rangs serrés. Si quelque chose se bloque quelque part, alors cette partie de la culture prend du retard. Quand on regarde le Japon dans son ensemble, on constate que la ligne de progression de la culture est en dents de scie: la capitale et la campagne évoluent de façons très différentes. Si bien que, même en l'absence de documents écrits, en rassemblant et en comparant aujourd'hui les choses qui se sont ainsi "figées ", on peut comprendre les évolutions qu'a connues le Japon. Dans des endroits tels que les plaines de Sibérie ou des États-Unis par exemple, on peut considérer peut-être qu'il existe simplement une avancée ordonnée et un front qui marque l'arrêt des éléments centraux de la culture, mais dans le cas d'un pays montagneux aussi divers que le Japon, à chaque avancée on tombe sur un cul-de-sac. C'est ce que l'on constate entre Tōkyō et la frontière du Shinsh $\bar{u}^{19}$ par exemple, où sont préservées des formes culturelles très anciennes. Ainsi, au Japon, on peut utiliser de façon extrêmement fructueuse les différences culturelles actuelles pour retracer une histoire qui s'affranchisse des documents écrits. Il n'existe d'ailleurs aucun autre pays qui possède, comme le Japon, plusieurs centaines d'îles qui ont chacune une culture singulière. En ce sens, la comparaison de lieux très éloignés entre eux sur le territoire japonais est tout particulièrement significative. Nous usons de cette méthode très fréquemment. Nous parcourons tout le Japon pour en faire l'analyse. Nous sommes ainsi en contact pour notre étude avec des personnes de toutes les régions du Japon. On oppose souvent le Nord-Est du pays au Sud-Ouest, mais en réalité les formes culturelles des deux régions se ressemblent énormément. Certes on remarque des différences ponctuelles, qui sont fonction du contexte environnant, mais finalement elles se ressemblent, car elles sont toutes les deux des régions éloignées du centre de plusieurs centaines de lieues. C'est la même chose, encore, avec une partie de la péninsule de Noto et le Kishü ${ }^{20}$.

SHIKIBA : En procédant de la sorte, où les études folkloriques cherchent-elles à aboutir?

Yanagita : Nous cherchons à faire de l'histoire du Japon une science moderne. Quelle que soit la méthode que nous adoptons, ce que nous cherchons c'est à déterminer le passé avec exactitude. C'est essentiellement sur ce point que nous sommes en débat avec les "sciences historiques", qui ne se contentent que des sources écrites. En effet, nous parvenons à comprendre des choses qui, jusqu'ici, étaient restées incomprises. Bien sûr, on pourrait dire que ce que nous faisons actuellement ne relève pas à proprement parler d'une " science $"^{21}$. Mais s'il arrive un jour que le mot « science » prenne un sens rigoureusement défini, alors nous sommes tout à fait prêts à abandonner le nom de "sciences folkloriques». Nous ne verrions alors aucun inconvénient à utiliser le terme d' « histoire » (shi 史) ou d' « archivisme» (shi 誌) $)^{22}$.

\section{Différences entre " arts populaires » et " études folkloriques »}

SHIKıвA : Nous avons pu entrevoir, grâce à vos explications, professeur Yanagita, ce qu'étaient les études folkloriques. Pour elles, si je comprends bien, en prenant par exemple la question de la langue, recueillir les dialectes propres à chaque région permet, en considérant les mots qui présentent la forme la plus ancienne parmi ces dialectes, de 
découvrir la forme ancienne des mots de notre langue... Ou alors faut-il penser qu'elles ont pour objectif concret d'agir vis-à-vis de ce que nous appelons le japonais, de compléter ces langues régionales, et de renforcer encore la langue japonaise actuelle elle-même? Finalement, les études folkloriques sont-elles un savoir qui se donne pour but de connaître le passé, ou bien un savoir tourné vers le présent et l'avenir?

Yanagita : Les études folkloriques constituent une discipline qui vise évidemment à rendre compte avec exactitude du passé. Pour cette raison, le futur ne fait pas partie de notre champ d'étude. Je discute il est vrai souvent de l'avenir de notre langue, mais ce n'est pas en tant que folkloriste que je le fais. Je suis en effet à la fois un folkloriste et un homme du commun. En un mot, c'est comme patriote que je parle de ces choses. Les études folkloriques peuvent certes apporter de l'eau à mon moulin, mais ce n'est pas pour autant que parler du devenir du japonais fait partie des objectifs des études folkloriques. Pour cette raison, du point de vue de mon travail, le fait de m'exprimer sur des débats de politique linguistique risque de créer des confusions $^{23}$.

SHıкıвA : Ainsi, il n'y a pas, dans les études folkloriques, d'implications directes impliquant une prise de position vis-à-vis de la culture, n'est-ce pas?

Yanagita : C'est exact ${ }^{24}$. Il est vrai que les historiens aujourd'hui débattent parfois de l'avenir ou de la façon d'appréhender l'homme moderne, mais cela n'entre pas, selon nous, dans le programme de l'histoire conçue comme science. Les historiens d'aujourd'hui sont également des patriotes et ont, à ce titre, le désir de participer au débat politique, mais ils ne font alors qu'instrumentaliser le savoir de l'histoire. Il nous paraît à nous suffisant de décrire les faits avec exactitude.

YANAgI : Les études folkloriques existent donc en tant que science empirique ${ }^{25}$ ?

SHוKıBA : Voilà donc la grande distinction entre le mouvement des Arts populaires et les études folkloriques. Qu'en pensez-vous professeur Yanagi?

Yanagi : Il me semble que le mouvement des Arts populaires correspond à une science normative ${ }^{26}$ plutôt qu'empirique. La question n'est pas de savoir tout ce qui existe, ou a existé : notre mission est au contraire d'aborder le monde de tout ce qui doit exister. Sur ce point, la différence entre arts populaires et études folkloriques est claire.

Yanagita : Excessivement claire, oui. Nous n'avons aucunement cette approche.

Yanagi : Ainsi, le mouvement des Arts populaires se rapproche de l'esthétique et accorde une grande importance au débat sur la valeur intrinsèque des choses.

Yanagita : J'imagine en effet que c'est ce à quoi vous arrivez.

SHIкıвA : Quels sont les rapports entre les études folkloriques et l'archéologie?

Yanagita : Pour proposer une distinction claire, je pense que l'on peut dire que la différence se situe essentiellement au niveau de leur objet, immatériel ou matériel. La question est donc: y a-t-il, ou pas, une chose tangible à étudier ? Ainsi, les arts populaires qui vous intéressent me paraissent pencher plutôt du côté de l'archéologie puisqu'ils traitent du matériel.

Yanagi : Cela est vrai, mais uniquement pour l'aspect du traitement des objets.

Yanagita : Nous, nous rassemblons des photographies et des esquisses d'objets réels, mais uniquement comme support, et nous ne les utilisons que comme illustrations lorsque les mots ne suffisent pas. 
SHIKıBA : À ce sujet, professeur Yanagi, quels rapports entretiennent le mouvement des Arts populaires et l'archéologie?

Yanagi : Les disciplines se ressemblent en effet en ce qu'elles se doivent, l'une et l'autre, d'observer de très nombreux objets. Mais il me semble qu'on ne peut dire de l'archéologie qu'elle est une science normative. L'archéologie ne se soucie pas non plus de discuter de la valeur des choses.

Yanagita : Cependant, il y a des points de ressemblance entre l'archéologie et l'histoire de l'art. Si l'on regarde dans le passé, l'intérêt pour les textiles en est un exemple parmi d'autres. Les tenants de l'archéologie ont été pris de passion pour le sujet, et espèrent que les textiles pourront être préservés, autant que possible, dans l'avenir. Cependant, l'archéologie ne se proclame certainement pas science normative, contrairement à vous en effet.

Yanagi : Pour le mouvement des Arts populaires la question du futur ne peut jamais être évacuée.

YANAgITA : Cependant, permettez-moi de vous poser la question: pensez-vous qu'une époque viendra à laquelle les anciens objets d'artisanat rassemblés ici, à la Maison des arts populaires, pourront engendrer un nouvel art populaire?

Yanagi : Nous croyons que, tôt ou tard dans le futur, de nouveaux objets relevant des arts populaires vont apparaître, ou encore, que les objets populaires anciens possèdent quelque chose qui doit apparaître. Voilà pourquoi nous militons pour le mouvement des Arts populaires.

\section{Points communs entre " arts populaires » et « études folkloriques »}

Shikiba : Nous avons bien perçu je pense les distinctions entre la nature empirique des études folkloriques du professeur Yanagita et le caractère normatif des arts populaires du professeur Yanagi. Cependant, malgré les différences de points de vue des études folkloriques, qui examinent le passé, et des arts populaires, qui s'inquiètent également de l'avenir, il existe, dans l'objet d'étude même choisi par chacune des disciplines, des points communs assez évidents. En premier lieu, les arts populaires et les études folkloriques ne sont plus des savoirs qui se concentrent uniquement sur la culture de l'aristocratie, mais ils laissent une place essentielle à la culture partagée par les masses ${ }^{27}$. Parallèlement, ils reconnaissent l'un et l'autre les particularités régionales ${ }^{28}$.

Yanagita : Exactement. Et j'ajouterai encore une chose. Nous pensons également que les gens d'autrefois étaient bien plus intelligents que ce que l'on croit aujourd'hui. L'intelligence, ce n'est pas simplement être lettré par exemple. Il y avait chez eux une clairvoyance. Ce fait, les personnes impliquées dans le mouvement des Arts populaires le disent tout comme nous. De nombreuses personnes restent dans l'idée que les hommes qui nous ont précédés étaient inférieurs à l'homme contemporain, et, imaginant que c'était particulièrement le cas des paysans, jugent un peu péremptoirement toutes sortes de choses. On peut avoir de la clairvoyance sans posséder l'écriture. Leur mémoire était tout particulièrement remarquable. En fait les humains ont perdu leur capacité de mémoire en même temps qu'ils apprenaient à lire. Les gens d'autrefois possédaient en tout cas des talents exceptionnels. 
Yanagi : En effet, nous ressentons également les choses ainsi. Il est un fait qui m'a frappé au sujet de la nourriture lors d'un de mes voyages récents dans le Tōhoku ${ }^{29}$. Les gens de Tōkyō imaginent souvent que les gens de la campagne n'ont rien de bon à se mettre sous la dent, mais c'est loin d'être le cas. Lorsque je suis allé à Aomori, j'ai demandé à goûter à des légumes marinés de la région [tsukemono]. Quelqu'un a alors parlé des légumes qu'on préparait chez lui. Il m'en a apporté une douzaine de variétés, emballées dans une pièce d'étoffe... Et tous étaient délicieux! J'adore les tsukemono, et je suis assez exigeant chez moi, mais je n'en ai jamais plus d'une ou deux variétés à disposition.

Yanagita: Le plus souvent, la nourriture de la campagne est d'une extrême simplicité. Il n'y a que quelques jours dans l'année où la nourriture sort de l'ordinaire. C'est pourquoi les gens se réjouissent particulièrement ces jours-là. Mais il existe bien d'autres occasions de se réjouir encore. Comme lorsque l'on porte pour la première fois un kimono fraîchement tissé par exemple. Dans ces moments, on peut l'imaginer, la joie est extrême. Or c'est cette joie qu'ont évoqué profondément pour moi les objets de la Maison des arts populaires que je viens de voir.

Yanagi : C'est cela. Nous exposons actuellement à la Maison des arts populaires des harnachements de chevaux... On disait, lorsque l'on utilisait un nouvel harnais, que le cheval lui-même se réjouissait. Bien sûr, ce n'était peut-être que l'imagination du conducteur du cheval, très heureux lui aussi... mais la joie et la satisfaction d'une femme qui voyait un homme porter le kimono qu'elle avait tissé devait être un sentiment très fort dans une vie humaine je pense.

Shikiba : Découvrir dans ces choses la valeur qu'y accordaient les hommes est donc un élément qui réunit les arts populaires et les études folkloriques!

\section{Concernant l'avenir des arts populaires}

SHIKıвA : Professeur Yanagi, je souhaiterais vous entendre un peu plus sur votre voyage dans le Tōhoku.

\section{YANAgITA : Où êtes-vous donc allé ?}

Yanagi : Je me suis rendu dans les six départements du Tōhoku. Je n'y ai passé qu'un mois environ, mais les circonstances étaient extrêmement intéressantes. Mon voyage était organisé par le Laboratoire sur les dégâts de la neige du département de Yamagata, qui avait mis sur pied une exposition de nouvelles productions d'art populaire dans chaque département. J'y étais en tant que conseiller, pour discuter des possibilités pour l'avenir. À Yamagata, avec lequel le laboratoire a évidemment plus de liens, il n'y avait non pas une mais trois expositions, en trois lieux différents, qui réunissaient beaucoup de choses, dont de très nombreux objets dont nous ne connaissions pas encore l'existence. Du fait des relations étroites du laboratoire avec le ministère de l'Agriculture, la plupart des objets, parmi lesquels beaucoup d'objets en bois ou en bambou, étaient naturellement liés à l'activité agricole, et provenaient principalement de la région du Shōnai ${ }^{30}$. Nous avons pu tenir une rencontre autour des rites calendaires et je pense que l'année prochaine nous serons en mesure de présenter une beaucoup plus grande variété d'objets. J'aimerais par la suite organiser une grande manifestation au magasin Mitsukoshi de Tōkyō par exemple, et je pense que ce sera une excellente occasion pour découvrir ce qui existe au Japon. 
Shikiba : Sans aucun doute.

Yanagi : Sur le plan de la langue, j'imagine que cette région doit poser de sérieuses questions à Monsieur Yanagita, mais pour nous, le Tōhoku est sans doute le lieu le plus riche en artisanat populaire. Nous pensons qu'il ne faut pas laisser mourir ces arts, mais au contraire les vivifier dans l'avenir, en préservant leurs spécificités régionales, et en faire une source de revenus pour les villages du Tōhoku.

Yanagita : Comme vous le dites, il y a de nombreux artisans dans la région du Tōhoku, mais avec cette tendance à produire de plus en plus des articles de piètre qualité.

Yanagi : C'est en effet de plus en plus le cas.

YANAgITA : Et il semble bien que les produits de qualité ne refassent surface que lors des expositions...

Yanagi : Cette tendance existe. La majeure partie de ces expositions était toutefois composée de pièces réellement utilisées aujourd'hui, qui témoignent que des techniques absolument admirables sont toujours conservées.

Yanagita : Que du fait de la situation économique, comme d'ailleurs de l'organisation du travail, de tels objets soient de moins en moins soigneusement fabriqués me paraît inévitable, mais il me semblerait regrettable que disparaissent vraiment les artisans les plus doués.

Yanagi : Il y a pourtant, dans ce que j'ai vu, un grand renouveau et une production florissante. De plus nous pouvons ne pas réserver ces objets à un usage campagnard, mais inciter à leur utilisation en ville également. Je pense qu'il est absolument nécessaire de réfléchir à fabriquer des objets utilisables dans la vie quotidienne actuelle.

Yanagita : Voilà qui paraitt toutefois fort difficile...

Shikiba : Je suis allé récemment à Sendai ${ }^{31}$, et en voyant l'exposition organisée là-bas, il m'a semblé qu'il y avait de nombreux objets que l'on pourrait introduire dans la vie quotidienne de nos contemporains.

Yanagi : Je place de grands espoirs dans la pantoufle ${ }^{32} \ldots$

YANAgITA : Ne craignez-vous que cette commercialisation, pour laquelle il existe une demande comme vous le disiez, ne soit possible que pour des objets utilisables tout au long de l'année?

Yanagi : Cela dépend vraiment de la direction que l'on veut donner aux affaires. Prenons l'exemple de ces coiffes appelées nizo. Elles sont produites dans la région d'Aizu. Une commande de 3000 pièces vient de parvenir des États-Unis. Elles seraient, semble-t-il, utilisées comme chapeau de plage en été. Ainsi, si nous arrivons à nous adapter à la demande, un réel potentiel existe.

Shikiba : Je pense qu'il n'est pas nécessaire pour les Arts populaires de faire vivre l'ensemble de ces objets anciens. Il suffit de répondre avec de l'artisanat ancien à la seule partie de ce qui est nécessaire au monde contemporain. Nous venons d'évoquer les pantoufles : il n'est aucunement obligatoire de préserver indéfiniment le concept de pantoufle tel qu'il a été importé d'Europe. Quand on y songe, il y a au Japon bien des exemples de technique de tressage pour des objets en paille qui pourraient tout à fait convenir à la fabrication des pantoufles. Il faut, dans ces conditions, commencer 
par rappeler l'existence de l'artisanat japonais. C'est alors que s'ouvrira la possibilité que le concept de pantoufle à l'occidentale aboutisse à des pantoufles japonaises, conçues avec une technologie japonaise. Le mouvement des Arts populaires souhaite de cette manière rendre la culture japonaise plus proprement japonaise, c'est un mouvement qui cherche à renforcer la culture japonaise. À cette fin, nous devons d'abord éduquer notre regard pour qu'il soit attentif aux arts populaires, car c'est ainsi que nous pourrons sélectionner des objets d'artisanats exceptionnels et variés, en qualité d'expert justement. Il nous suffit ensuite de faire confiance : à chacun de trouver des voies pour donner vie à ceux-ci dans la vie quotidienne actuelle.

Yanagita : Voilà une position bien compréhensible.

Shikiba : Récemment, un architecte nouvelle mode nous a rendu visite à la Maison des arts populaires et il a reçu un choc en découvrant différents tissus d'Okinawa, qu'il disait absolument magnifiques et tout à fait utilisables pour la décoration intérieure.

Yanagi : Nous sommes absolument convaincus que les objets possédant cette beauté véritable que permet l'artisanat ne perdent jamais, quel que soit le temps qui passe, leur valeur de nouveauté. Quand on les regarde de nouveau, nombreux sont les motifs parmi les anciens tissus kasuri d'Okinawa qui possèdent une très grande fraîcheur ${ }^{33}$. Il y a également des choses très surprenantes dans ces expositions du Tōhoku dont nous parlions. Il serait vraiment regrettable, pour l'ensemble de la culture japonaise elle-même, que ces objets, qui existent bel et bien, ne suscitent aucun attachement, et soient finalement rejetés, sous prétexte que toute chose est destinée à changer.

Yanagita : Si un artisanat populaire au contenu renouvelé est en effet possible, il faut évidemment l'enseigner.

Yanagi : Je pense par ailleurs que certaines choses peuvent connaître une évolution en suivant cet esprit. Prenons l'exemple des soieries de Yūki ${ }^{34}$. Ces produits, très chers, ne sont pas destinés au grand public, mais comme ils possèdent une réputation de qualité, ils trouvent toujours acheteurs, quelle que soit la production ou si élevé que soit leur prix. Vendre beaucoup et à bas prix est une stratégie, une autre, et hautement admirable, est de ne pas craindre de vendre cher des objets qui en valent la peine.

Yanagita : Il est possible d'enrayer le déclin de l'artisanat pour peu que des gens compréhensifs s'en occupent un peu et le soutiennent. Cependant, les machines progressent à une vitesse effroyable et l'on peut craindre qu'un jour ou l'autre les objets artisanaux eux-mêmes ne soient fabriqués de façon industrielle.

SHIKIBA : Cependant, et j'adresse ma question au professeur Yanagi, les avancées de la machine lui permettront-elles de rendre l'aspect « fait main »?

Yanagi : On doit pouvoir l'imiter en tout cas...

Yanagita : La différence n'est pas perceptible au premier regard !

Yanagi : Cependant, et quoi qu'il en soit, les produits artisanaux et industriels diffèrent dans leur essence. Je crois que la valeur de l'artisanat est, quoi que l'on fasse, inégalable. 
Yanagita : Sur les céramiques également, les décors sont exécutés par des machines. On s'y laisse prendre parfois...

Yanagi : Le mouvement des Arts populaires s'oppose, en un certain sens, à la machine, mais je pense qu'il suffit de travailler en complémentarité, en proposant un monde que les machines ne peuvent posséder. Il est vrai que nous choisissons de nombreux objets d'artisanat anciens, mais ce n'est pas simplement parce qu'il s'agit d'objets anciens, ou d'artisanat, mais parce que nombreux sont les beaux objets qui relèvent de l'ancien artisanat. Nous ne voulons pas non plus nous borner à dire que ces objets sont beaux, mais nous essayons de comprendre pourquoi ils sont beaux, et comment ils sont devenus beaux. Puis, comme nous pensons qu'il nous faudra dans l'avenir aussi fabriquer des choses finalement belles, nous analysons les critères du beau à l'œuvre dans ces choses anciennes, et nous réfléchissons à la façon de faire vivre ces objets anciens dans le futur. Tel est, je pense, le grand travail auquel nous nous sommes attelés ${ }^{35}$.

\section{NOTES}

1. Shikiba et Yoshida, regrettant la faible diffusion de la revue Artisanat (Kōgei 工藝) dirigée par Yanagi, proposent la publication d'un mensuel militant et engagé à large diffusion pour répandre «l'esthétique mingei » auprès du public. Dans l'éditorial du premier numéro du Mensuel, Shikiba présente celui-ci comme la mitraillette (kikanjū 機関銃), soit «l'arme de diffusion massive »du mouvement. Yanagi, pourtant connu pour son pacifisme et son antimilitarisme, semble ne pas s'offusquer de l'emploi d'un tel vocabulaire et offre de financer la revue par le biais de l'Association pour les arts populaires. L'entretien que nous proposons de traduire ici est justement publié dans le Mensuel.

2. Toutes les notes sont des traducteurs.

3. Traduction de 《Mingei to minzokugaku no mondai » 民藝と民俗學の問題 (La question des arts populaires et des études folkloriques), Gekkan Mingei, vol. 2, nº 4, avril 1940 ; YSZ 10, p. 735-747.

4. Yanagi mobilise ici, sans doute par ignorance des termes en usage à son époque, un terme ancien, dozokugaku 土俗学. D’abord utilisé au début de Meiji pour désigner le domaine de l'anthropologie qui s'occupe des artéfacts culturels, celui-ci va rapidement avoir pour sens l'étude des marges du Japon, puis des ethnies autres, des aborigènes. Il est remplacé par le terme minzokugaku 民族学 à partir des années 1920, et disparaît alors. On comprend le peu d'empressement que Yanagita met à lui répondre, et la nécessité ressentie par Shikiba de redéfinir les termes, quelques lignes plus bas. Nous avons choisi de traduire dozoku par " autochtone " par référence commune à la terre (do).

5. Esunorojīエスノロジー, de l'anglais ethnology.

6. Minzoku 民族.

7. C'est-à-dire de peuples non développés, parmi lesquels il est impossible de compter les Japonais pour Yanagita.

8. Comme l'explique Yanagita, le terme qui s'est imposé pour parler des études folkloriques (folk studies) est problématique car homonyme de celui désignant l'ethnologie. On ne peut faire la différence qu'en considérant les caractères chinois utilisés: dans un cas zoku désigne les 
« coutumes » 俗 (du «peuple »: min), dans l'autre les «familles» ou les « lignées» 族 ( famille du peuple»= ethnie). On passe donc de l'intérêt pour les pratiques sociales à l'idée que l'appartenance à un groupe donné (potentiellement : une race) est essentielle dans la définition de l'identité. L'opposition dozoku/minzoku (avec le même zoku) est d'un autre ordre. L'intérêt premier pour le do, la terre, le local, l'autochtone finalement, est abandonné dans le premier quart du $\mathrm{xx}^{\mathrm{e}}$ siècle, écrasé par la montée en puissance de la nation et de la notion de "peuple ». C'est d'ailleurs ce même peuple qui lie, dans leurs appellations comme dans leur projet et leurs enjeux, arts populaires (min-gei) et science des coutumes du peuple, ou études fokloriques (minzoku gaku).

9. Genshi minzoku 原始民俗.

10. Respectivement : mikaijin 未開人, hankaijin 半開人.

11. Shizengaku 史前学. Ōyama Kashiwa (1889-1969), chercheur qui a alterné travaux scientifiques et participation à l'expansion militaire (il a été capitaine de l'armée de terre mais a également enseigné à Keiō), est surtout connu pour sa participation à l'archéologie des années 20 et 30 et ses travaux sur la période Jōmon. Il fonde, chez lui, le «Laboratoire d'études préhistoriques » en 1928. Ce dernier disparaitra dans les bombardements américains de mai 1945.

12. Taishitsu jinruigaku 体質人類学 et bunka jinruigaku 文化人類学.

13. Kōkogaku 考古学.

14. En réalité Yanagita est plus attiré par la langue que par la linguistique. Les études folkloriques vont établir ainsi de nombreux lexiques listant, par thème (le mariage, la pêche...), les différents termes régionaux.

15. Mingei 民藝.

16. En anglais dans le texte.

17. L'Attic Museum se donnait en effet pour but de recueillir les outils «populaires » (mingu 民

具) dans leur diversité afin de faire une étude soignée des différentes techniques de construction et d'utilisation ; voir l'article de Damien Kunik dans ce numéro.

18. Utsukushii tadashii mingeihin.

19. Partie montagneuse (alpes japonaises) au centre de Honshū, le Shinshū correspond à l'actuel département de Nagano.

20. Noto (département d'Ishikawa) est une péninsule qui pointe dans la mer du Japon en partant du milieu de Honshū à peu près. Le Kishū correspond à la pointe sud-est de la péninsule de Wakayama, dans le Pacifique. Ce sont donc deux endroits symétriquement opposés quand on prend pour axe Kyōto-Tōkyō. Également distants des capitales, au fond d'une péninsule, ces deux «pays» ne peuvent que se ressembler, pensent les folkloristes. Yanagita était persuadé que s'éloigner de la capitale c'était effectuer une remontée dans le passé japonais. Il considérait très logiquement que les plus vieux restes de l'antique civilisation japonaise étaient encore visibles à Okinawa, ou chez les Aïnus, et préconisait l'analyse des marges japonaises pour comprendre le vrai sens des traditions anciennes. Cette attitude nie évidemment la capacité d'évolution propre à chaque territoire, de même qu'elle fait fi de l'établissement des réseaux (administratifs, religieux, commerciaux, familiaux, etc.) qui participèrent, chacun à leur manière au cours de l'histoire japonaise, aux transformations des lieux qu'ils reliaient. En tout état de cause, l'idée d'une progression concentrique de la culture à partir d'un grand centre - la capitale impériale n'est plus considérée comme une hypothèse sérieuse aujourd'hui.

21. Gaku 学, que nous avons choisi de traduire par «études» par référence à l'anglais studies, mais qui peut aussi se comprendre comme « science ", comme le dit Yanagita dans cette phrase.

22. Yanagita utilise le caractère employé comme équivalent $\mathrm{du}$ "-graphie » d'ethnographie. Il a longtemps milité pour la pratique du minzoku-shi, soit la discipline qui se donne pour tâche de relever et de décrire les coutumes du peuple (d'où notre "archivisme» ici), dans le but de permettre des comparaisons régionales. Shi évoque plus littéralement l'idée de prendre note. 
23. Yanagita prend ses précautions en prévision du débat sur la politique linguistique du Japon à Okinawa qui clôt cet entretien (partie non traduite).

24. Cette affirmation est curieuse : Yanagita voulait en effet faire des études folkloriques un savoir utile à son époque, en tentant, à travers des enquêtes dites "d'urgence » (kinkyū chōsa 緊 急調査) de retrouver les pratiques ayant cimenté la société japonaise que l'urbanisation était en train de mettre à mal. Elle ne se comprend donc que dans le cadre strict de ce dialogue.

25. Keikengaku 経験学.

26. Kihangaku 規範学. Yanagi emploie le mot posé comme équivalent de l'allemand norm depuis 1922.

27. Taishū-sei 大衆性, la « nature » partagée par les masses.

28. Chihō-sei, le « caractère régional ».

29. Région au nord-est de Honshū.

30. Région du Tōhoku qui correspond grosso modo à la partie du département de Yamagata donnant sur la mer du Japon.

31. Préfecture du département de Miyagi, dans la région du Tōhoku.

32. La question avait été discutée par les deux hommes hors entretien.

33. Le kasuri est un type de "double ikat » : les fils de trame et de chaîne teints préalablement à des endroits précis sur leur longueur forment un motif lorsqu'ils sont tissés ensemble. On retrouve cette technique particulièrement dans les Ryūkyū.

34. Région à l'ouest du département d'Ibaraki.

35. L'entretien se poursuit en évoquant la question de la politique linguistique japonaise à Okinawa. Ce passage concernant peu le rapport entre mingei et minzoku gaku, nous nous permettons de terminer cette traduction sur cette phrase de Yanagi.

\section{RÉSUMÉS}

Débat entre Yanagi Sōetsu et Yanagita Kunio sur la nature de leurs discipline et méthodes respectives, à la Maison des arts populaires (1940).

Discussion between Yanagi Sōetsu and Yanagita Kunio on their own discipline and methods, at the Japan Folk Crafts Museum (1940).

\section{INDEX}

Keywords : Anthropology, Ethnology, Folk Art, Material Culture, Mingei, Popular Culture, Utensils, mingu, Yanagi Sōetsu (1889-1961), Archeology

キーワード : minshū geijutsu 民衆芸術, taishū bunka 大衆文化, mingei undō 民芸運動, mingu 民具, Yanagi Sōetsu 柳宗悦 (1889-1961), minzoku 民俗, minzokugaku 民俗学, jinruigaku 人類学, minzokugaku 民族学, kōkogaku 考古学, Yanagita Kunio 柳田國男 (1875-1962), Okinawa 沖縄, Shōwa jidai 昭和時代 (1923-1945), Shōwa jidai 昭和時代 (1945-1989)

Mots-clés : arts populaires, culture populaire, mingei - mouvement artistique, outils, Yanagi Sōetsu (1889-1961), folklore, études folkloriques

Thèmes : anthropologie, ethnologie, archeology

Index chronologique : Shōwa (1923-1945), Shōwa (1945-1989) 